\title{
Réfugiés et migrants en Afrique : quel statut pour quelle vulnérabilité ?
}

Luc Cambrézy

\section{(2) OpenEdition}

1 Journals

Édition électronique

URL : https://journals.openedition.org/remi/4199

DOI : $10.4000 /$ remi.4199

ISSN : $1777-5418$

Éditeur

Université de Poitiers

Édition imprimée

Date de publication : 1 décembre 2007

Pagination : 13-28

ISBN : 978-2-911627-47-4

ISSN : 0765-0752

Référence électronique

Luc Cambrézy, «Réfugiés et migrants en Afrique : quel statut pour quelle vulnérabilité ? », Revue européenne des migrations internationales [En ligne], vol. 23 - $n^{\circ} 3$ | 2007, mis en ligne le 01 décembre 2010, consulté le 14 avril 2022. URL : http://journals.openedition.org/remi/4199 ; DOI : https://doi.org/ 10.4000/remi.4199

Ce document a été généré automatiquement le 14 avril 2022.

() Université de Poitiers 


\title{
Réfugiés et migrants en Afrique : quel statut pour quelle vulnérabilité?
}

\author{
Luc Cambrézy
}

1 Alors qu'à l'origine, les migrations internationales du Sud vers le Nord ont été largement impulsées par les anciennes puissances coloniales en manque de maind'œuvre ${ }^{1}$, la fin des «trente glorieuses » au tournant des années 1970 a totalement renversé la perspective. Les pays industrialisés, et la France tout particulièrement, ont depuis mis en place des mesures sans cesse plus restrictives pour «maîtriser les flux migratoires » originaires des pays du Sud. En quelques décennies, nous sommes donc passés d'une immigration encouragée à une immigration que le gouvernement français actuel n'hésite pas à qualifier de «subie ». Dès lors, la thèse est inversée : il ne s'agit plus de répondre, par l'immigration, aux nécessités économiques des pays industrialisés du Nord, mais de contrôler une migration désormais interprétée, au Nord, comme une migration de la misère; en d'autres termes, une réponse «spontanée » des populations des pays du Sud confrontées à la pauvreté et au sousdéveloppement. Et de fait, l'actualité sans cesse plus médiatisée participe de manière quasi quotidienne à cette "démonstration " en rendant compte des épreuves et des drames qui jalonnent le parcours des migrants qui, venant du Sud, tentent de pénétrer en Europe. Selon cette analyse, la vulnérabilité serait donc le corollaire de la pauvreté et du sous-développement et on comprend le succès que rencontre la politique du « codéveloppement " présentée désormais comme la solution censée permettre de limiter, voire de stopper, les migrations Sud - Nord.

Dans le cadre de cette réflexion ${ }^{2}$, nous proposons d'envisager la question de la vulnérabilité au regard d'une des formes particulières de la migration internationale : celle des populations réfugiées issues des nombreux conflits qui ont frappé le continent africain au cours des dernières décennies. Après un bref rappel des flux de population que génèrent les conflits, seront ensuite successivement analysées les formes de vulnérabilité auxquelles ces populations sont exposées; d'une part dans les pays dont 
ils sont originaires, et, d'autre part, dans les différents contextes qu'ils rencontrent ensuite au cours de leurs itinéraires migratoires. Dans un second temps, cette analyse sera mise en perspective au regard de deux autres types de migration: d'une part la population rurale africaine dont la fragilité des systèmes agraires menace sans cesse l'équilibre population/ressources; d'autre part, les « réfugiés de l'environnement », un groupe qui n'a pas de réalité formelle mais qu'un nombre croissant d'analystes s'attache à construire en tant que catégorie. À partir de cette double mise en perspective, sera discutée pour conclure la question de l'élargissement du droit d'asile des réfugiés à d'autres groupes de population.

\section{La protection des réfugiés en Afrique : quelle vulnérabilité ?}

3 La notion de vulnérabilité est au cœur de la définition du statut de réfugié tel que celuici est précisé en droit international. Selon la convention de Genève de 1951, est réfugié toute personne qui, «craignant avec raison d'être persécutée du fait de sa race, de sa religion, de sa nationalité, de son appartenance à un certain groupe social ou de ses opinions politiques, se trouve hors du pays dont elle a la nationalité et qui ne peut ou, $\mathrm{du}$ fait de cette crainte, ne veut se réclamer de la protection de ce pays » (article $1^{\mathrm{er}} \mathrm{A} / 2$ de la Convention de Genève). En d'autres termes, la vulnérabilité est ici à interpréter comme étant le risque d'être persécuté et, par extension, de craindre pour sa vie ou celle de ses proches. Mais avec une importante réserve. Le risque ne s'applique qu'à des situations bien précises : il faut avoir quitté son pays pour des raisons qui sont toutes immatérielles (religion, appartenance, opinons politiques, etc.). En négatif et a priori, sont donc exclues du statut de réfugié les personnes qui ont quitté leur pays d'origine pour d'autres raisons que celles citées. On ne devient pas réfugié parce qu'on a quitté un pays ravagé par une sécheresse, une épidémie ou une crise économique. Enfin la définition du réfugié est strictement dépendante du tracé des frontières et donc de la carte politique du monde et de la liste des États reconnus comme tels par l'ONU. ${ }^{3} \mathrm{Au}$ total, la problématique de la vulnérabilité chez les réfugiés renvoie d'abord à la question de l'appartenance et à l'identité. Elle est consubstantielle de politiques et de pratiques discriminatoires qui visent certaines catégories particulières de population vivant au mauvais endroit et appartenant - en période de crise - au "mauvais côté ». Il n'est en revanche pas fait référence à la situation de très grande vulnérabilité économique et sociale qui prévaut pourtant dans le contexte des conflits armés.

De cette ambiguïté naissent de nombreuses controverses lorsque la question de l'immigration de travail en Europe se superpose, sans se confondre, à celle de la demande d'asile des réfugiés. On comprend dès lors que, selon les acteurs, les interprétations soient largement divergentes. Les administrations des États du Nord s'acharnent à débusquer les "faux réfugiés " et tentent de restreindre le champ d'application des conventions internationales; les organisations humanitaires, les chercheurs et les instances internationales sont partagés entre, d'une part, le strict respect du cadre juridique défini par la protection des droits de l'homme et, de l'autre, l'élargissement du statut de réfugié à diverses catégories de migrants. Quant aux populations concernées elles-mêmes, selon les lieux et les circonstances, elles peuvent aussi bien vivre le statut de réfugié comme une humiliation, une contrainte ou au contraire comme leur seule porte de salut (Cambrézy, 2006). 
5 Par ailleurs, si la convention de Genève constitue le cadre juridique dans lequel s'inscrit la définition du statut de réfugié, elle ne définit pas pour autant clairement les modalités d'application de la protection de cette catégorie de population. En étant muette sur les procédures de reconnaissance du statut, elle laisse toute liberté d'interprétation aux États. Dès lors, en fonction des pays et des contextes politiques, économiques et sociaux, la protection effective des victimes des conflits est sujette à une interprétation extrêmement large qui, souvent, ne contribue guère à diminuer la situation de vulnérabilité initiale des populations contraintes de fuir les violences et les conflits. Ainsi, dans les pays les plus pauvres, en Afrique en particulier, la protection précaire des réfugiés alimente de nouvelles mobilités. Ces migrations en chaine sont motivées par la quête de solutions durables et viables à des problèmes que l'assistance humanitaire est rarement en mesure de résoudre de manière satisfaisante et que l'on peut résumer en deux facteurs clés qui sont liés : le droit à un statut juridique digne de ce nom et le « droit d'habiter ».

6 Dans le cas des guerres civiles et des conflits armés, la fuite et l'exil des populations déplacées ou réfugiées apparaissent moins comme une "réponse » que comme une urgence imposée par la nécessité de survie. Les migrants forcés ne quittent pas leur pays ou leur région d'origine dans l'espoir d'un avenir meilleur. Au contraire, en fuyant vers l'inconnu, ces populations s'exposent dans la plupart des cas à une vulnérabilité accrue et à l'incertitude du retour. Ceci étant dit, on remarquera au passage que cela ne permet guère de trancher entre la pertinence ou non d'une distinction entre migration de travail (ou migration économique) et migration forcée. Il est de ce fait sans doute plus utile de s'intéresser aux formes concrètes de vulnérabilité qui affectent les populations réfugiées et déplacées en raison des conflits armés dont ils sont victimes.

\section{En Afrique, la protection précaire des réfugiés}

7 Si nous pouvions nous satisfaire des statistiques, il y aurait lieu de se réjouir. En effet, depuis la situation catastrophique qui prévalait durant les années 1990, le nombre de réfugiés a très sensiblement diminué. Fin 2005, on comptait 8,4 millions de réfugiés dans le monde, soit l'effectif le plus bas depuis 1980. En Afrique, les 2,6 millions de réfugiés enregistrés en 2005 sont à comparer aux 6,7 millions de 1994. L'évolution plutôt encourageante ainsi enregistrée depuis quelques années devrait donc conduire à l'optimisme. Pourtant, cette vision résiste mal à l'analyse. En effet et sans préjuger de l'avenir, même si plusieurs conflits semblent appartenir au passé - au Rwanda, en Angola, au Libéria, ou au Sierra Leone,... (parfois par la mort ou l'exil de l'un des protagonistes de ces guerres: Jonas Savimbi en Angola, Charles Taylor au Libéria) -, d'autres crises apparaissent (Darfour) ou ne cessent de rebondir à mesure que certains conflits s'internationalisent (Somalie, Sud-Soudan,...). D'autre part, s'en tenir à la stricte définition du réfugié telle qu'elle est inscrite dans le droit international ne peut faire oublier les millions de déplacés ${ }^{4}$ qui, pour diverses raisons, n'ont pas pu ou voulu quitter leur pays d'origine et vivent souvent des situations largement aussi précaires que les réfugiés ayant franchi une frontière. Or nombre de guerres civiles "produisent" autant - sinon plus - de déplacés (sans statuts) que de réfugiés («statutaires»). Pour peu que ces populations soient soupçonnées d'appartenance au «mauvais côté », ce n'est pas même la question du droit d'asile qui se pose à ces populations que celle de leur survie. Et cette question est d'autant plus grave que les parties en conflit (gouvernement ou opposition) s'emploient souvent à contrecarrer 
l'action conduite par les organisations humanitaires pour assurer la protection de ces personnes 5 .

Mais d'autres éléments de cadrage s'imposent également. Rappelons tout d'abord que l'immense majorité des réfugiés issus des conflits en Afrique reste cantonnée en Afrique et se trouve massivement concentrée dans les pays limitrophes des pays en guerre où la population obtient le statut de réfugié sur une base collective (prima facie). Pour autant, cela ne va pas nécessairement dans le sens d'une meilleure protection. Car, si la convention de 1969 de l'organisation de l'Union Africaine (OUA) élargit en effet le statut de réfugié "sans qu'il soit nécessaire de démontrer une persécution individuelle " il reste que "cet élargissement de la notion de réfugié participe du phénomène de déresponsabilisation des États occidentaux face aux crises qui ne les touchent pas directement » (Rodier, 2002). Ainsi, alors que $80 \%$ des réfugiés en Afrique ont obtenu le statut sur la base collective ou prima facie, le contraste est frappant avec l'Europe ou la majorité (voire la totalité) des demandeurs a obtenu le statut de réfugié sur une base individuelle. Et le HCR de conclure: "Les différences régionales en matière de reconnaissance s'expliquent en partie par la nature du cadre juridique existant ainsi que par le niveau de développement économique (la détermination individuelle du statut de réfugié est exigeante en termes de ressources)» (UNHCR $2005: 6$ ).

Dans les pays africains de premier accueil, si les proximités linguistiques et culturelles jouent parfois en faveur d'une assez bonne disposition des populations locales à l'égard des populations réfugiées, il ne s'agit là que d'exceptions qui confirment la règle générale. Comme ailleurs, l'hospitalité a ses limites et du point de vue des pays d'accueil, l'afflux des réfugiés fait plus souvent craindre le pire en termes d'insécurité, d'exportation du conflit au-delà des frontières, et de dégradation de l'environnement (accès au bois de feu, à l'eau, etc.). Enfin, autant par réticence que par incapacité matérielle et financière à gérer l'urgence et à déployer les moyens logistiques nécessaires, les États africains font largement appel au Haut Commissariat aux Réfugiés pour assurer l'accueil et la protection des réfugiés; tout en négociant âprement - souveraineté oblige - les modalités d'intervention des organisations humanitaires. De ce fait, la protection des réfugiés se trouve ainsi placée sous une sorte de double tutelle: celle, très largement subordonnée à l'aide délivrée par la communauté internationale par le biais du HCR, mais aussi celle du bon vouloir (à géométrie variable) de l'État d'accueil. Du point de vue institutionnel et politique, les divergences d'intérêts et d'objectifs, les ambiguïtés de toute nature et le rejet mutuel des responsabilités en cas de litige - constituent l'arrière-plan de toute négociation entre un État souverain et les Nations unies (en l'occurrence, le HCR). Dès lors, dire que les conditions d'une protection satisfaisante des réfugiés ne sont pas remplies relève à l'évidence de l'euphémisme.

10 Par ailleurs, tous les réfugiés ne bénéficient pas des mêmes attentions. Il va de soi que la protection dont bénéficient de fait les présidents déchus, les anciens chefs de guerre en déroute et leurs suites, est sans rapport avec le drame vécu par les centaines de milliers de civils fuyant les zones de combat. Pour les premiers, la négociation se joue directement avec les gouvernements des pays amis et, jusqu'à une période récente, ils pouvaient vivre un exil discret et tranquille avec leur famille et leurs fidèles, sans être autrement inquiétés par d'éventuelles poursuites judiciaires ${ }^{6}$. À l'inverse, ces cas particuliers mis à part, la grande majorité des réfugiés se trouve regroupée dans des 
camps. En général aménagés à proximité des frontières de leur pays d'origine, ces camps - censés être temporaires - durent ce que durent les conflits, c'est-à-dire plusieurs années, voire, plusieurs décennies. Les camps fonctionnent selon une double logique - l'une sécuritaire, l'autre humanitaire - dont il faut souligner la cohérence de la "mécanique ». D'une part, dans la limite du possible, il s'agit de contrôler les mouvements des réfugiés de manière à éviter leur dispersion dans le pays d'accueil ; d'autre part, on s'attache à répondre dans ces camps aux besoins les plus vitaux de ces populations (abri, santé, alimentation, eau). La seconde fonction agit donc sur la première. Répondre aux besoins de ces populations est une façon de les faire rester là où l'on souhaite les maintenir.

11 Cependant, les recommandations du HCR, issues des expériences passées, visent un principe d'équité. Au nom de celui-ci, il est dit et répété qu'il serait contre-productif (et cause de tensions supplémentaires) d'offrir aux réfugiés des conditions générales d'existence supérieures au quotidien vécu par la population locale. De ce fait - on le comprend bien eu égard aux conditions générales de vie des populations rurales en Afrique - ce principe autorise et justifie un nivellement par le bas du volume et de la qualité de l'aide qui peut à tout instant menacer le fragile équilibre entre logique d'assistance et logique de contrôle des populations. Situés dans des environnements souvent médiocres et parfois même exposés à des risques divers (naturels et sécuritaires), les camps offrent à leurs occupants une vie aux termes plus que comptés : distribution parcimonieuse de l'eau et des rations alimentaires, abris rudimentaires, propreté et hygiène souvent menacées, sécurité incertaine... Les interdits y sont aussi plus nombreux que les droits. Les opportunités de travail sont rares quand celui-ci n'est pas simplement prohibé et l'agriculture, l'élevage ou l'accès aux ressources en bois de feu sont des domaines strictement soumis au bon vouloir de la population locale et des autorités gouvernementales. En d'autres termes, malgré tous les efforts des organisations humanitaires, on finit par se contenter en Afrique de cette sorte de « service minimum » comme s'il pouvait tenir lieu de politique d'asile.

\section{Du statut de réfugié à celui de clandestin : les conséquences de la précarité}

Dans le cas des conflits et des mouvements de personnes qui s'ensuivent, la problématique de la vulnérabilité n'est pas seulement une question de droit et de protection des minorités et identités menacées du fait de leur appartenance ethnique ou de leurs convictions religieuses ou politiques. À l'analyse, on s'aperçoit aussi qu'elle est aussi à « démographie et géographie variable ». Par exemple, il y a encore quelques années, chaque nouvelle crise humanitaire liée à des situations de conflit était l'occasion pour les organisations en charge de l'assistance aux réfugiés de préciser à l'intention des médias que les victimes étaient «majoritairement des femmes et des enfants ». Même si cette approximation statistique visant à amplifier le sentiment de compassion a été depuis corrigée ${ }^{7}$, cette formule résume bien l'impuissance de l'aide humanitaire à traiter de manière satisfaisante le problème des réfugiés dans son ensemble. Cette impuissance explique la politique consistant à introduire de nouvelles catégories permettant de cibler des strates particulières de population jugées justement plus «vulnérables » que les autres: les femmes et les enfants, considérées comme plus fragiles que les hommes; les femmes seules, plus que les femmes mariées; 
les enfants «non accompagnés ", plus que les enfants encadrés par des adultes; les vieillards, les infirmes... ${ }^{8}$.

Par ailleurs, la durée des conflits - conjuguée à la politique d'endiguement des réfugiés dans des camps - joue incontestablement contre une politique d'asile digne de ce nom. Conséquence de l'enlisement de nombre de conflits, il arrive que des réfugiés passent dix, quinze ou vingt ans dans des camps et ce, parfois dans plusieurs pays successifs. Or les camps sont le lieu de fréquents accès de violence dus notamment à une aide qui ne correspond pas aux attentes des réfugiés. Cela vaut d'abord pour les distributions alimentaires dont la réduction échauffe rapidement les esprits, mais cela vaut également pour d'autres aspects de la vie quotidienne ; à commencer par un constant et insupportable sentiment d'humiliation lui-même généré et entretenu par cette logique d'assistance et d'oisiveté forcée? .

Dans ce contexte, les réfugiés les moins démunis ou socialement les mieux armés choisissent d'échapper aux camps en choisissant de se fondre dans l'anonymat des grandes villes. Mais dans cet autre environnement, toute la question est de savoir si les réfugiés y sont tolérés ou non par le gouvernement du pays d'accueil. Dans le meilleur des cas - mais il s'agit d'une minorité - ils peuvent bénéficier du statut de réfugié, voire, d'un permis de travail. Dans le pire, ces réfugiés sans statut - et donc « hors la loi » aux yeux du gouvernement - subissent les habituelles conséquences de la clandestinité : travail précaire et non réglementé, scolarisation aléatoire des enfants, assistance médicale hasardeuse et coûteuse, harcèlements policiers, déménagements répétés, dénonciations, emprisonnements sans protection judiciaire, (Cambrézy, 1997). Ainsi se pose, pour cette catégorie de réfugiés, une question dont on ne voit pas l'issue. Pour ces « réfugiés de fait ", mais sans statuts ni protection, la durée de l'exil conduit à une errance qui brouille les repères et les catégories. Partis d'Afrique centrale, on les retrouve des années plus tard au Sénégal ou en Afrique du Sud, en Belgique ou en Grande-Bretagne. Et c'est alors de nouveau la question de leur statut juridique, en Europe cette fois, qui se trouve posée. En se lançant dans des aventures et des périples comparables à ce que vivent les migrants économiques en quête de travail, comment peuvent-ils encore faire valoir leurs droits ? Et en ont-ils encore?

\section{Mobilités et migrations en milieu rural : une réponse ancienne à la fragilité des systèmes agraires}

Dans les sociétés rurales africaines, l'émigration vers un ailleurs plus ou moins lointain représente souvent pour tout ou partie de la population la solution qui s'impose lorsque la satisfaction des besoins essentiels se trouve menacée. Cette migration n'est d'ailleurs pas toujours aussi dramatiquement vécue qu'on ne le pense. Tout dépend des circonstances et du lieu de la nouvelle installation. La distance géographique et sociale entre le lieu de départ et de destination joue en la matière un rôle prépondérant qu'on ne doit pas sous-estimer. Les difficultés rencontrées comme les manières de répondre aux situations de crise sont diverses. Mais les raisons de migrer s'articulent globalement autour d'une situation générale de pauvreté et de sous-développement liée pour une large part à un niveau de production insuffisant pour répondre aux besoins les plus élémentaires (alimentation, santé, éducation), mais aussi à d'autres aspirations moins "essentielles" comme par exemple l'accès à des produits de consommation courante. Ce sous-développement trouve ses causes profondes dans la 
faible productivité des systèmes agraires qui, dans un contexte de pression démographique et foncière croissante, ne peuvent évoluer in situ sans l'introduction d'investissements (matériels et immatériels) massifs qui ne peuvent venir que de l'extérieur. Dans le cas des systèmes de production davantage ouverts sur les cultures d'exportation que sur l'autosubsistance, il faut ajouter la forte dépendance de ces économies vis-à-vis de l'évolution des cours mondiaux des produits agricoles; notamment lorsque ces derniers sont concurrencés par les productions subventionnées des agricultures du nord (coton, céréales...). Dans ce contexte global de crise du monde rural, ici décrite à très grands traits - parce que toute bouche à nourrir désœuvrée constitue une charge difficilement supportable - une partie de la population doit donc envisager de migrer ; au pire, pour diminuer le poids de cette charge ; au mieux, pour espérer aider davantage ceux qui restent grâce aux envois d'argent. D'une certaine manière donc, on peut dire que pour nombre de sociétés africaines, la nécessité de migrer s'inscrit dans le contexte de crise de systèmes en tension perpétuelle et ce, sans qu'il soit nécessaire d'invoquer des événements plus ou moins imprévus et exceptionnels tels que les catastrophes naturelles (sécheresse) ou les conflits politiques. En d'autres termes, le cas le plus général est celui, apparemment paradoxal, où la situation de crise devient ou est devenu au fil du temps «la situation normale » à laquelle il convient de s'adapter en envisageant un départ.

16 Partir donc, telle est la réponse, mais il y a plusieurs manières de le faire. Lorsque la situation de pauvreté chronique est étroitement corrélée à la pression démographique et foncière, les sociétés rurales africaines ont une manière simple et efficace d'y répondre. Une partie de la population - souvent les générations montantes - se déplace des régions les plus peuplées vers celles encore vides ou moins peuplées. On observe ce phénomène dans des pays et des régions aux conditions agro-écologiques aussi différentes que le Niger, le Rwanda ou le nord de l'Ouganda (Cambrézy 2006). Dans ces trois cas, alors même que les conditions climatiques et les systèmes de production agricoles n'autorisent pas des charges de population comparables, le même processus de colonisation agricole en tache d'huile a pu être observé. Il se traduit par un double phénomène conduisant à une densification lente ou nulle dans les régions d'origine les plus peuplées et accélérée dans les régions périphériques de destination. $\mathrm{Au}$ total, on assiste donc à une extension progressive de l'espace peuplé (et exploité) par un double processus de densification et de redistribution de la population. Mais nécessairement, dans un contexte de forte croissance démographique, cette forme de réponse trouve un jour ou l'autre ses limites. L'espace réellement utilisable est d'autant moins extensible que certaines parties du territoire national sont inaptes à l'agriculture (précipitations insuffisantes, sols dégradés), trop éloignées ou situées trop à l'écart des régions les plus dynamiques pour bénéficier des infrastructures suffisantes et être réellement attractives. Les régions semi-arides vouées au pastoralisme - faute d'investissement permettant par exemple une agriculture irriguée - sont emblématiques de cette situation d'isolement condamnant à court terme toute perspective de développement. L'espace, pourtant largement disponible, ne constitue pas alors en soi une ressource suffisante pour retenir la population ou en attirer de nouvelles.

17 C'est pourquoi, parallèlement ou suite à la conquête de nouvelles terres, d'autres stratégies sont également employées. L'exode rural et la croissance urbaine (davantage étudiée d'ailleurs que le processus de densification et de dispersion précédemment 
décrit), sont souvent présentés comme la traduction d'une évolution inéluctable qui conduirait l'Afrique subsaharienne à suivre, même avec retard, le modèle des pays industrialisés où le taux d'urbanisation atteint des sommets. Il est vrai cependant que la barre symbolique des $50 \%$ de la population vivant en ville sera prochainement atteinte (UNFPA, 2007). Pourtant, au-delà des problèmes de mesure et de définition entre « rural » et " urbain », « l'explosion urbaine » - et l'expression n'est évidemment pas neutre - ne doit pas faire oublier que si la population des villes ne cesse de croître, la population rurale ne diminue pour l'instant qu'en valeur relative mais continue d'augmenter en valeur absolue et ce, dans la plupart des pays d'Afrique subsaharienne ; ce qui ne fait d'ailleurs que confirmer le processus général décrit précédemment de peuplement continu et élargi de l'espace rural. D'autre part, l'exode rural n'a pas pour seul effet de faire gonfler de manière spectaculaire la population des capitales. Plus discrètement, ce sont aussi les villes petites et moyennes, et les bourgs en situation de carrefour économique (les trading centers en Afrique anglophone) qui voient leurs effectifs augmenter dans d'importantes proportions. Ces localités constituent d'ailleurs souvent des étapes dans l'histoire migratoire des individus dont les rythmes (temporaires, saisonniers ou "définitifs») sont largement fonction des lieux de destination. Enfin, si l'exode rural est bien une réponse aux difficultés que rencontrent les sociétés agraires, le rythme élevé de la croissance urbaine et le caractère limité du marché du travail ne garantissent pas que l'installation de nouveaux migrants en ville soit couronnée de succès. C'est d'ailleurs pourquoi la croissance urbaine n'est pas un phénomène à sens unique en Afrique puisque dans certaines circonstances on assiste au processus inverse d'abandon des villes et de "retour" à la campagne (Beauchemin, 2002, 2005).

$18 \mathrm{Au}$ total, on retiendra surtout qu'en milieu rural, la mobilité et les migrations doivent être probablement perçues davantage comme une réponse normale à la fragilité des systèmes agraires. D'une certaine manière, contrairement à l'idée qui prévaut encore parfois de sociétés immuables, enracinées et voire, "sans histoire ", la mobilité et la migration font partie intégrante des systèmes agraires. De ce fait la distinction formelle établie par les milieux académiques entre migrations internationales et migrations dites «internes» pose d'autant plus question que l'on tend - s'agissant des premières - à circonscrire le sens de cette expression aux seuls mouvements de population du Sud vers le Nord. En d'autres termes, le franchissement de la frontière politique d'un État serait d'autant plus problématique (et donc, digne d'intérêt) que la distance géographique et sociale entre la population locale et la population migrante serait élevée. Dès lors, les appartenances nationales mises ainsi en contact diraient à elles seules le «sentiment»-plus ou moins partagé par le pays et la population d'accueil - qu'inspire l'arrivée de ces migrants. Ces derniers sont-ils les bienvenus ? Sont-ils jugés utiles, voire, nécessaires? Ces appréciations, qui valent autant pour les migrants économiques que pour les réfugiés, ne sont d'ailleurs pas gravées dans le marbre; on l'a d'ailleurs vu au cours de la décennie passée lorsque la population burkinabé installée depuis l'époque coloniale en Côte d'Ivoire s'est vue brutalement rejetée au nom de «l'ivoirité ».

\section{Crise ou catastrophe? Les « réfugiés de l'environnement »}

19 À l'aune de ce bref rappel des conditions qui prévalent dans les sociétés rurales africaines, la question de ce que d'aucuns dénomment les «réfugiés de 
l'environnement " prend une dimension d'autant plus intéressante qu'il est rare de mettre en perspective ces deux groupes de population. Rappelons les conditions dans lesquelles ce débat s'est instauré.

Depuis de nombreuses années, un mouvement plus ou moins organisé d'acteurs divers entend élargir la notion de réfugié aux victimes des catastrophes naturelles. Ces initiatives, conduites par des organisations de protection environnementale, mais aussi des chercheurs, sont corrélées dans le temps avec la prise de conscience croissante de la responsabilité de l'homme dans la dégradation des milieux et, aujourd'hui, dans le réchauffement climatique. Ce mouvement ne survient d'ailleurs pas de manière anodine puisqu'il correspond à un moment de l'histoire où s'installe dans les esprits l'idée que protection de l'environnement et prévention des conflits sont deux objectifs étroitement liés ${ }^{10}$. Les arguments avancés laissent cependant planer quelques interrogations sur ces prises de position. En effet, la reconnaissance juridique du statut de "réfugié de l'environnement» serait censée donner accès à une assistance humanitaire dont les victimes des catastrophes naturelles sont supposées être dépourvues (Gonin et Lassailly-Jacob, 2002). Or cette affirmation s'avère discutable. Depuis déjà de nombreuses années, les effets dévastateurs des séismes, des éruptions volcaniques, des inondations ou des sécheresses suscitent une émotion largement comparable à celle que provoquent les conflits en Afrique dont les causes, elles, sont souvent mal comprises. Depuis longtemps, les organisations internationales, les ONG mais aussi les États, proposent leur aide - et notamment, l'aide d'urgence - aux pays victimes de ces catastrophes. On se souvient d'ailleurs du coup d'éclat de l'organisation "Médecins Sans Frontières » à la suite du Tsunami qui toucha les côtes thaillandaises du 26 décembre 2004 (280 000 victimes). L'avalanche d'aides venues de toute part incita l'ONG à demander de cesser l'envoi de dons et de mieux coordonner les actions... au grand dam des autres organisations humanitaires.

Du côté des organisations environnementales, scientifiques et militants écologistes ont, dès le début des années 1980, pris soin d'attirer l'attention des opinions publiques sur les conséquences sur les populations des dégradations de la nature liées à l'action anthropique. Ils pariaient avec raison sur le fait que les opinions publiques et les gouvernements seraient d'autant plus sensibles aux problèmes environnementaux que les risques pour les sociétés humaines seraient aisément compréhensibles. Il fallait donc montrer les victimes des catastrophes. Or, d'une part, ce sont incontestablement les populations des pays les plus pauvres qui souffrent le plus durement des conséquences des excès de la nature: mauvaise qualité des constructions ou constructions dans des sites à haut risque (d'inondations, de glissements de terrain, etc.), infrastructures de secours et de prévention des risques insuffisantes ou inexistantes. D'autre part, l'impact médiatique des images est d'autant plus fort que les populations affectées sont nombreuses. Cela justifie-t-il pour autant d'élargir la définition du réfugié aux victimes de ce type de catastrophes? Le rappel d'un événement survenu à l'occasion du cyclone Katrina (29 août 2005) peut aider à y voir plus clair.

On s'en souvient, les conséquences de cette catastrophe firent l'objet d'une couverture médiatique à la hauteur de l'événement. Et, à l'instar de leurs confrères américains de CNN ou de Fox News, les journalistes du monde entier adoptèrent rapidement le terme de «réfugiés " pour désigner les sans-abri victimes du cyclone. Jusqu'à ce qu'une soudaine polémique conduise progressivement à remplacer ce terme par "sinistrés » 
ou «déplacés». Ironie de l'histoire, cette querelle sémantique, jugée d'abord avec légèreté, devint - parce qu'il s'agissait de la communauté noire - une question politique d'une extrême sensibilité. C'est dans la population noire, principale victime du déluge et des inondations, que naquit cette controverse lorsque le révérend Jesse Jackson déclara qu'il serait "raciste de qualifier des citoyens américains de réfugiés " (Le Monde du 7 septembre 2005) en regrettant par ailleurs que les victimes du cyclone puissent être perçues comme "des citoyens de seconde zone». Au-delà de la supposée "connotation péjorative" que le pasteur prêtait à ce terme, il est un fait que la définition du réfugié telle qu'elle est inscrite dans la convention de Genève de 1951 donnait raison aux victimes (noires ou blanches) du cyclone. Même si les populations sinistrées de La Nouvelle-Orléans contraintes de quitter leur domicile ont dû chercher refuge ailleurs, cela n'en faisait pas pour autant des réfugiés. Dans le cas du cyclone Katrina, tout indique que cet élargissement du sens ne s'expliquait pas seulement par une sorte de facilité ou de paresse de langage que se seraient accordés les médias, la presse audiovisuelle en particulier. En fait, même si d'autres termes existent pour cela, le mot « réfugié » est porteur d'une charge émotionnelle et dramatique forte qui «fait» de l'audience. Comme dans bien d'autres catastrophes, la surenchère dans la mise en scène de ce drame humanitaire explique cette inflation langagière.

Les milieux académiques et/ou associatifs spécialisés dans les questions environnementales seraient-ils également touchés par cette dérive médiatique ? C'est bien sûr la question que pose l'insistance mentionnée plus haut à vouloir parler de " réfugiés de l'environnement " pour désigner les victimes des catastrophes naturelles. Pour tout dire, cette hypothèse parait d'autant plus envisageable que, comme les crises humanitaires, les catastrophes environnementales sont à fort potentiel médiatique. Plus largement, le milieu concerné est évidemment sensible aux importantes sources de financement associées à l'action humanitaire et à l'environnement, deux activités qu'il faut bien prendre pour ce qu'elles sont: deux marchés. Dès lors, au risque d'une terminologie racoleuse, les conditions objectives sont effectivement réunies pour céder à la tentation de la surenchère médiatique. Le cyclone Katrina constitue une opportunité parmi d'autres pour ceux qui cherchent à consolider ce rapprochement entre l'action humanitaire à destination des populations réfugiées et celle destinée aux victimes des catastrophes naturelles. Volontairement ou non, les médias ont servi cette cause, mais, malheureusement pour ceux qui la soutiennent, l'objection est venue des victimes elles-mêmes.

Depuis cet «incident sémantique » (de fait, rapidement oublié) force est de constater que le débat reste d'actualité et on ne compte plus les articles en faveur d'un élargissement du concept de réfugié à la problématique de l'environnement. Il est vrai que la prise de conscience croissante d'une planète menacée par le réchauffement climatique et les désordres qui l'accompagnent y contribue largement. Ainsi, dans son édition d'avril 2007, Le Monde diplomatique n'hésitait-il pas à titrer : "au Bangladesh, les premiers réfugiés climatiques»(Garnier, 2007). Mais ce contexte brouille le jugement plus qu'il ne le clarifie. Tout événement climatique ou environnemental d'ampleur tend à devenir par l'amplification médiatique une " catastrophe »; au risque d'oublier que la nature est riche d'événements majeurs plus ou moins cycliques qui sont moins liés à l'action anthropique qu'aux conditions physiques et géographiques qui permettent qu'ils se manifestent. Pour autant, ne nous trompons pas de débat. Il ne consiste évidemment pas à contester la dimension politique de la problématique environnementale. Car il est rare que les dégâts provoqués par la Nature ne soient pas 
partiellement ou entièrement associés à une responsabilité humaine. Même dans le cas du cyclone Katrina (entretien des digues), mais plus encore dans les pays du Sud, on sait combien les victimes « doivent " aux lacunes de la prévention, aux constructions non conformes et aux habitations situées dans des zones non constructibles, bref, à l'impéritie de l'État.

Mais ce rappel étant fait, le retour à la question des sociétés rurales africaines doit ramener le débat à sa juste dimension. L'une des grandes fragilités des systèmes agraires africains tient justement à son extrême dépendance à l'égard des incertitudes climatiques. Que les pluies soient excessives, trop tardives ou notoirement insuffisantes, les conséquences sont attendues : des récoltes trop maigres ou dévastées promettent aux populations concernées des jours sombres. De même, une succession d'années sèches dans les zones pastorales semi-arides se traduit inévitablement par une mortalité élevée du cheptel. Ces risques et ces incertitudes sont connus depuis tous temps par les populations. Dès lors, les questions qui s'imposent mais que ne posent pourtant pas les partisans du concept de "réfugié de l'environnement" sont les suivantes : les agriculteurs africains ne sont-ils pas tous un jour ou l'autre des réfugiés de l'environnement? Et s'il fallait répondre par l'affirmative, peut-on être assuré que les populations concernées tireraient un réel avantage de ce nouveau statut? Dans des systèmes de production fragiles et si étroitement dépendants des déséquilibres environnementaux qu'aucune mesure préventive ou de protection ne permet de corriger, quel sens faut-il donner au terme de "catastrophe »? N'est-il pas de la responsabilité du chercheur en sciences sociales de conserver une distance vigilante au regard de l'extraordinaire médiatisation des questions environnementales?

\section{Élargissement du statut de réfugié et respect du droit d'asile, une équation à hauts risques}

On l'a vu, il existe de nombreuses raisons conduisant au fait que des dizaines de milliers de personnes se retrouvent hors de leur pays, sans statut migratoire ni protection, malgré les circonstances qui devraient pourtant leur permettre d'accéder au statut de réfugié. Le plus souvent parce qu'ils ne sont pas là où l'on juge qu'ils devraient être (dans le pays de première arrivée, dans des camps, etc.), toute cette population passe ainsi progressivement du statut de réfugié de droit, à celui de fait, puis à celui - si on peut dire - d'illégal et de clandestin. Au nord comme au Sud, cette population tend à se confondre avec la situation vécue par les migrants économiques. Mais l'insuffisante prise en compte des problèmes économiques et sociaux vécus par les pays et les populations en guerre ou en situation de post-conflit conduit paradoxalement à augmenter le nombre de réfugiés sans statut sur la voie de la migration économique et alimente ainsi les flux migratoires mixtes. Ces flux, composés de "vrais réfugiés sans statut » et de migrants économiques revendiquant le statut de réfugié, ouvre le champ à de si nombreuses interprétations que la mauvaise foi devient la qualité la mieux partagée.

Dans ce contexte, poser la question du droit d'asile en Europe conduit inévitablement à se poser aussi celle de l'asile en Afrique. On l'a vu à propos des camps de réfugiés, l'asile en Afrique, est un peu le service minimum et l'on voit bien que cette question ne peut être dissociée de celle, plus englobante, du respect des droits de l'homme au regard de la réalité de l'État en Afrique. En d'autres termes, travailler pour un plein respect des 
droits d'asile en Europe ne peut conduire à se satisfaire des conditions de l'asile en Afrique. Le chantier est d'autant plus urgent qu'il concerne des millions de personnes et que l'on a sans doute trop longtemps cru que l'endiguement des réfugiés dans des camps pouvait constituer le meilleur rempart contre la dispersion incontrôlée de ces populations. Il est clair que mieux la protection des réfugiés sera assurée en Afrique, mieux le droit d'asile en Europe sera respecté.

En matière de politiques migratoires, on constate que la mondialisation s'accompagne de tensions croissantes entre le Nord et le Sud. Dans ces conditions, la question de l'élargissement du statut de réfugié aux victimes des catastrophes environnementales - mais aussi, par extension, aux paysans victimes des caprices de la nature - a le mérite de soulever le problème fondamental du développement et d'une meilleure répartition des richesses comme des systèmes de protection. Mais inversement, le risque existe que le remède soit pire que le mal. En Afrique, la protection des réfugiés se heurte à deux obstacles majeurs : l'absence d'une politique à la fois volontariste et humaniste en matière de droit d'asile des réfugiés d'une part (Cambrézy et Laacher, 2007); la faiblesse des moyens dont disposent les organisations humanitaires pour aider ces pays à appliquer les termes des conventions internationales, d'autre part. Or, dans le contexte actuel, la situation n'est guère plus favorable aux réfugiés africains qui tentent d'atteindre le Nord de la Méditerranée. Pour l'heure, l'Europe - soucieuse d'une plus grande maîtrise des flux migratoires - se montre de plus en plus sourcilleuse dans l'attribution du droit d'asile aux réfugiés. Cette politique de fermeture se traduit par un double phénomène; d'une part, le statut de réfugiés de plein droit ne reste accordé qu'au compte-gouttes ${ }^{11}$; de l'autre, le nombre de demandes d'asile en Europe ne cesse de diminuer non pas tant en raison d'une pression plus faible que par le fait des différentes mesures restrictives destinées à «combattre l'immigration illégale et l'usage abusif du système du droit d'asile» (HCR, $2000: 161$ ). Ces mesures sont au nombre de quatre. C'est d'abord la politique dite de "non arrivée " empêchant les étrangers de pénétrer sur le sol européen. La seconde mesure est celle $\mathrm{du}$ " détournement »; elle consiste à transmettre les demandes d'asile et de protection à d'autres pays. La troisième mesure vise à privilégier une application restreinte de la convention de 1951 sur les réfugiés. Par exemple, en ne reconnaissant pas le statut de réfugiés à des victimes de persécution commises par des agents "non étatiques ${ }^{12}$. Au mieux, ces restrictions permettent de demeurer dans le pays où la demande d'asile a été déposée, mais se traduisent par « une reconnaissance réduite au minimum de leurs besoins de protection, en particulier en matière de regroupement familial et de délivrance de documents de voyage ». Enfin, les pays industrialisés recourent de plus en plus à des "mesures dissuasives", comme la mise en détention des demandeurs d'asile, le refus de toute aide sociale et la limitation de l'accès à l'emploi.

C'est donc au moment où les portes se ferment sur les "vrais réfugiés » que les partisans d'un élargissement du statut - portés par l'engouement que suscite la protection de la planète - sont les mieux entendus. Il y a là un paradoxe qu'il faut d'autant mieux mesurer que l'enjeu appelle à la responsabilité. On devine sans peine ce que la cause des demandeurs d'asile aurait à perdre à une telle banalisation de l'exclusion dont ils sont victimes. L'écologie politique (quelle que soit sa couleur) et la protection militante de l'environnement ne peuvent se construire en fragilisant la vigilance de tous les instants qu'exige la protection des droits de l'homme. 


\section{BIBLIOGRAPHIE}

BEAUCHEMIN Cris (2002) Des villes aux villages : l'essor de l'émigration urbaine en Côte d'Ivoire, Annales de géographie, $\mathrm{n}^{\circ}$ 624, pp.157-178.

BEAUCHEMIN Cris, SCHOUMAKER B. (2005) Migration to Cities in Burkina Faso : Does the Level of Development in Sending Areas Matter?, World Development, Vol. 33, No. 7, pp. 1129-1152.

CAMBREZY Luc (1997) Un aspect méconnu de la crise rwandaise : les réfugiés de Nairobi, Politique Africaine, $\mathrm{N}^{\circ} 68$, pp. 134-141.

CAMBREZY Luc (2006) Territoire et dimension géopolitique de l'accueil des réfugiés. Les colonies agricoles des exilés du Soudan en Ouganda, Nature, Sciences, Sociétés, Vol. 14, Oct-Déc., pp. 365-375.

CAMBREZY Luc, LAACHER Smaïn (2007) L'asile au Sud: Afrique, Méditerranée, La Chronique, n 53. Paris, Ceped, pp. 1-4.

GARNIER Donatien (2007) Au Bangladesh, les premiers réfugies climatiques, Le Monde Diplomatique, avril 2007.

GONIN Patrick, LASSAILLY-JACOB Véronique (2002) Les réfugiés de l'environnement. Une nouvelle catégorie de migrants forcés ?, Revue européenne des migrations internationales, vol. 18, $\mathrm{n}^{\circ}$ 2, pp. 139-160.

HCR (2000) Les réfugiés dans le monde. Cinquante ans d'action humanitaire, Paris, Éditions Autrement, $338 \mathrm{p}$.

HCR (2005) Réfugiés : tendances mondiales en 2005. UNHCR, Genève, 115 p.

http://unhcr.ch/include/fckeditor/custom/File/PublicStatist/Statistiques/

TendancesMondiales_f.pdf

RODIER Claire (2002) La construction d'une politique européenne de l'asile entre discours et pratiques, Hommes et Migrations, $\mathrm{n}^{\circ} 1240$.

UNFPA (2008) Libérer le potentiel de la croissance urbaine. État de la population mondiale 2007, NewYork, $99 \mathrm{p}$.

\section{NOTES}

1. Dans le cas de la France, il ne s'agissait pas seulement d'une immigration en direction de la métropole. Le développement des cultures de plantation (café, cacao) a également conduit le colonisateur à favoriser des mouvements de population "internationaux » à l'intérieur même de l'empire colonial ; par exemple la migration mossi de l'ex Haute Volta en direction de la Côte d'ivoire.

2. Insistons sur le fait qu'il s'agit davantage ici d'une mise en perspective construite sur la base de plusieurs programmes de recherche conduits en Afrique, que de la restitution proprement dite de résultats nouveaux.

3. Avec cependant quelques cas très particuliers (réfugiés palestiniens, sahraouis, kosovars,...) liés à l'incertitude juridique du territoire disputé.

4. Pour des raisons très largement politiques (non ingérence, souveraineté des États,...), la question de la protection des personnes déplacées du fait des guerres civiles et des conflits interethniques à l'intérieur d'un même pays fait l'objet - de la part de la «communauté 
internationale »- d'une attention moindre que celle des réfugiés. Cela mérite d'être rappelé au regard de la controverse relative aux « réfugiés de l'environnement » qui sera analysée plus loin. 5. Nombre de guerres civiles passées ou actuelles illustrent ce problème : Angola, RDC, Soudan, Ouganda.

6. Il faut souligner la montée en puissance de la justice internationale. Pour ne citer que lui, parmi les dictateurs disparus, Idi Amin Dada, l'ex-président de l'Ouganda aurait aujourd'hui du souci à se faire... En Europe, Slobodan Milosevic, l'ancien président yougoslave, est mort en prison à La Haye. C'est également en Hollande que Charles Taylor (ex-président du Liberia) devrait être jugé par un Tribunal spécial pour certaines de ses exactions passées, tandis que le tchadien Hissène Habré se voit de plus en plus menacé dans son exil au Sénégal. Plusieurs hauts responsables du gouvernement Habyarimana attendent d'être jugés au tribunal d'Arusha en Tanzanie pour leur rôle joué dans le génocide rwandais. Cependant, pendant ce temps, Menguistu, l'ex-dictateur éthiopien, condamné par contumace, à la prison à vie par la Haute Cour fédérale de son pays, bénéficie de la protection du gouvernement du Zimbabwe.

7. En effet, quel que soit le sexe ratio, en mélangeant deux catégories distinctes (une classe d'âge et un genre), on obtient toujours un chiffre majoritaire. Pourtant, s'il peut exister au niveau local des différences importantes dans la répartition des sexes, il n'en est rien au niveau de l'ensemble de la population réfugiée en Afrique comme dans le monde : la répartition entre garçons et filles et hommes et femmes est conforme aux lois normales de la démographie.

8. Le HCR et les organisations humanitaires conduisent des actions spécifiques à l'endroit des "vulnerable groups " pour lesquels ils s'emploient à maintenir des statistiques aussi actualisées que possible.

9. La « réinstallation » dans un pays tiers est un autre motif de très vives tensions. Malgré les efforts du HCR pour accroître le nombre de places disponibles, la réinstallation n'est accessible qu'à une petite proportion de réfugiés dans le monde - en général les plus vulnérables. Par exemple, parmi les victimes du conflit en Somalie où les enjeux se mesurent à l'aune des hiérarchies dans l'appartenance à tel clan ou sous-clan, les «somali bantu», jugés plus «vulnérables » ont bénéficié d'un traitement privilégié.

10. Le prix Nobel de la Paix de 2007 que se partagent Al Gore et le groupe d'experts intergouvernemental sur l'évolution du climat (GIEC), lié à l'ONU, en est la plus récente démonstration.

11. En 2006, en France, seulement $18 \%$ des demandes d'asile ont été résolues par l'attribution du statut de réfugié. Les demandes d'asile ont chuté de $38 \%$ entre 2005 et 2006.Le Mondedu 22/09/07, p. 10.

12. Au niveau européen, ce motif de rejet a été ensuite abandonné en 2004.

\section{RÉSUMÉS}

Cet article envisage la notion de vulnérabilité dans le contexte précaire de l'accueil des réfugiés en Afrique. Après un bref rappel des chiffres, la situation des réfugiés sera mise en relation avec deux autres types de migrations. D'une part celles liées à la fragilité des sociétés rurales en Afrique; d'autre part, les migrations liées aux crises environnementales, qui, selon certains spécialistes, justifient d'élargir le statut de réfugié aux «réfugiés de l'environnement ». Cette dernière question sera discutée en conclusion dans le contexte européen d'une forte restriction du droit d'asile. 
Refugees and Migrants in Africa: which Status for which Vulnerability? The concept of vulnerability is analyzed in the precarious context of hosting refugee population in Africa. After a quick look at the data, the refugees' situation is considered in relation with two other types of migration. It is first compared with migrations caused by the fragility of rural communities in Africa; then with migrations related to the crisis of the environment that, following some experts, justify the new concept of "environmental refugees". Finally, this last question is discussed in the context of the strong tightening of asylum in Europe.

Refugiados y migrantes en Africa: que tipo de estatus para qual vulnerabilidad? Este articulo trata la nocion de vulnerabilidad en el marco muy fragil de la poblaciones refugiadas. Despues de un breve recuerdo de las estadisticas, la situacion de los refugiados estara estudiada en relacion con otros tipos de migraciones. Por un lado, las migraciones ligadas a la fragilidad de las sociedades rurales; por el otro lado, las migraciones debidas a diversas crisis del medio ambiente, quines, segun ciertos especialistas justifican que le estatus de refugiado sea extendido a los «refugiados del medio ambiente». Finalmente, este problema sera discutido en el contexto de una reduccion drastica del asileo en Europa.

\section{AUTEUR}

\section{LUC CAMBRÉZY}

Géographe, Directeur de Recherche à l'IRD. 32, avenue Henri Varagnat - 93143 Bondy cedex. 